\title{
Towers and Fibered Products of Model Structures
}

\author{
Javier J. Gutiérrez and Constanze Roitzheim
}

\begin{abstract}
Given a left Quillen presheaf of localized model structures, we study the homotopy limit model structure on the associated category of sections. We focus specifically on towers and fibered products (pullbacks) of model categories. As applications we consider Postnikov towers of model categories, chromatic towers of spectra and Bousfield arithmetic squares of spectra. For stable model categories, we show that the homotopy fiber of a stable left Bousfield localization is a stable right Bousfield localization.
\end{abstract}

Mathematics Subject Classification. 55P42, 55P60, 55S45.

Keywords. Localization, model category, Postnikov tower, homotopy fibered product, homotopy pullback.

\section{Introduction}

Localization techniques play an important role in modern homotopy theory. For several applications it is often useful to approximate a given space or spectrum by simpler ones by means of localization functors. For instance, given a simplicial set $X$, one can consider its Postnikov tower. This tower can be built as a sequence of fibrations

$$
\cdots \stackrel{f_{n}}{\longrightarrow} P_{n} X \stackrel{f_{n-1}}{\longrightarrow} P_{n-1} X \stackrel{f_{n-2}}{\longrightarrow} \cdots \stackrel{f_{2}}{\longrightarrow} P_{2} X \stackrel{f_{1}}{\longrightarrow} P_{1} X \stackrel{f_{0}}{\longrightarrow} P_{0} X
$$

and maps $p_{n}: X \rightarrow P_{n} X$ satisfying that $p_{n}=f_{n} \circ p_{n+1}$ for every $n \geq 0$ and that $\pi_{k}\left(f_{n}\right): \pi_{k}(X) \cong \pi_{k}\left(P_{n} X\right)$ if $k \leq n$ for any choice of base point of $X$, and $\pi_{k}\left(P_{n} X\right)=0$ if $k>n$ and all choices of base points.

Each of the spaces $P_{n} X$ can be built as a localization of $X$ with respect to the map $S^{n+1} \rightarrow *$, and $p_{n}$ is the corresponding localization map. If $X$ is connected, then the fiber of $f_{n-1}$ is an Eilenberg-Mac Lane space

J. J. Gutiérrez was supported by the NWO (SPI 61-638) and the MEC-FEDER Grants MTM2010-15831 and MTM2013-42178-P. Both authors received support from the LMS Scheme 4 Grant No. 41360. 
$K\left(\pi_{n}(X), n\right)$ and every simplicial set $X$ can be reconstructed as the homotopy limit of its Postnikov tower $X \simeq \operatorname{holim}_{n \geq 0} P_{n} X$; see [15, Chap. VI, Theorem 3.5].

In the category of spectra, given any spectrum $E$, we can consider its associated homological localization functor $L_{E}$ which inverts the maps that induce isomorphisms in $E_{*}$-homology in a universal way. Given an abelian group $G$, let us denote by $M G$ the associated Moore spectrum. It is well known that any spectrum $X$ can be built, using Bousfield's arithmetic square [9], as a homotopy pullback of the diagram of homological localizations

$$
L_{M \mathbb{Z}_{J}} X \longrightarrow L_{M \mathbb{Q}} X \longleftarrow L_{M \mathbb{Z}_{K}} X
$$

where $J$ and $K$ form any partition of the set of prime numbers and $\mathbb{Z}_{J}$ are the integers localized at the set of primes $J$.

Furthermore, the chromatic convergence theorem [26, Theorem 7.5.7] states that a finite $p$-local spectrum $X$ is the homotopy limit of its chromatic localizations $L_{E(n)} X$ at the prime $p$.

The aim of this paper is to present categorified versions of these statements in the framework of Quillen model structures. Given a diagram (left Quillen presheaf) of model categories $F: \mathcal{I}^{\text {op }} \rightarrow$ CAT, there is an injective model structure on the category of sections associated wit $F$, which we can further colocalize to obtain the homotopy limit model structure. We study these model structures for towers and homotopy fibered products (homotopy pullbacks) of model categories.

First, we construct the Postnikov tower of an arbitrary combinatorial model category. As an application we show that for simplicial sets and for bounded below chain complexes these towers converge in a certain sense. Another tower model structure is the homotopy limit model structure on the left Quillen presheaf of chromatic towers Chrom $(\mathrm{Sp})$, where Sp denotes here the category of $p$-local symmetric spectra. We show that the Quillen adjunction

$$
\text { const : Sp } \rightleftarrows \operatorname{Chrom}(\mathrm{Sp}): \lim
$$

induces a composite

$$
\mathrm{Ho}(\mathrm{Sp})^{\text {fin }} \stackrel{\mathbb{L} \text { const }}{\longrightarrow} \mathrm{Ho}(\mathrm{Chrom}(\mathrm{Sp}))^{F} \stackrel{\text { holim }}{\longrightarrow} \mathrm{Ho}(\mathrm{Sp})^{\text {fin }}
$$

which is isomorphic to the identity. (Here, $F$ and fin denote suitable finiteness conditions.) This set-up is a step towards deeper insights into the structure of the stable homotopy category via viewing chromatic convergence in a categorified manner.

We then move to fibered products of model categories. Using this setup, we show that the category of symmetric spectra is Quillen equivalent to the homotopy limit model structure of the left Quillen presheaf for Bousfield arithmetic squares of spectra.

As a final application we focus on a correspondence between the homotopy fiber of a left Bousfield localization $\mathcal{C} \rightarrow L_{\mathcal{S}} \mathcal{C}$ and certain right Bousfield localizations. This is then used, among other examples, to understand the 
layers of the Postnikov towers established earlier and to study the correspondence between stable localizations and stable colocalizations.

\section{Model Structures for Sections of Quillen Presheaves}

In this section we recall the injective model structure on the category of sections of diagrams of model categories. We will state the existence of this model structure in general, although we will be mainly interested in the cases of sections of towers and fibered products of model categories. Details about these model structures can be found in [4, Section 2, Application II], [6,7], [16, Section 3] and [27, Section 4].

Let $\mathcal{I}$ be a small category. A left Quillen presheaf on $\mathcal{I}$ is a presheaf of categories $F: \mathcal{I}^{\text {op }} \rightarrow$ CAT such that for every $i$ in $\mathcal{I}$ the category $F(i)$ has a model structure, and for every map $f: i \rightarrow j$ in $\mathcal{I}$ the induced functor $f^{*}: F(j) \rightarrow F(i)$ has a right adjoint and they form a Quillen pair.

Definition 1.1. A section of a left Quillen presheaf $F: \mathcal{I}^{\text {op }} \rightarrow$ CAT consists of a tuple $X=\left(X_{i}\right)_{i \in \mathcal{I}}$, where each $X_{i}$ is in $F(i)$, and, for every morphism $f: i \rightarrow j$ in $\mathcal{I}$, a morphism $\varphi_{f}: f^{*} X_{j} \rightarrow X_{i}$ in $F(i)$ such that the diagram

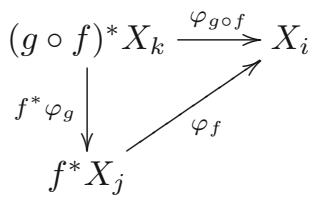

commutes for every pair of composable morphisms $f: i \rightarrow j$ and $g: j \rightarrow k$.

A morphism of sections $\phi:(X, \varphi) \rightarrow\left(Y, \varphi^{\prime}\right)$ is given by morphisms $\phi_{i}: X_{i} \rightarrow Y_{i}$ in $F(i)$ such that the diagram

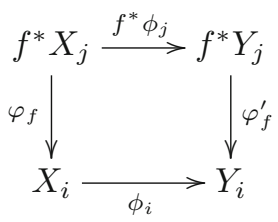

commutes for every morphism $f: i \rightarrow j$ in $\mathcal{I}$.

A section $(X, \varphi)$ is called homotopy cartesian if for every $f: i \rightarrow j$ the morphism $\varphi_{f}: f^{*} Q_{j} X_{j} \rightarrow X_{i}$ is a weak equivalence in $F(i)$, where $Q_{j}$ denotes a cofibrant replacement functor in $F(j)$.

Recall that a model category is left proper if pushouts of weak equivalences along cofibrations are weak equivalences, and right proper if pullbacks of weak equivalences along fibrations are weak equivalences. A model category is proper if it is left and right proper.

The category of sections admits an injective model structure, which is left or right proper, if the involved model structures are left or right proper, respectively. A proof of the following statement can be found in [4, Theorem 2.30, Propostion 2.31]. Recall that a model category is called combinatorial if it is cofibrantly generated and the underlying category is locally 
presentable. Foundations of the theory of combinatorial model categories may be found in $[5,11,23]$. The essentials of the theory of locally presentable categories can be found in $[1,14,24]$.

Theorem 1.2. (Barwick) Let $F: \mathcal{I}^{\mathrm{op}} \rightarrow$ CAT be a left Quillen presheaf such that $F(i)$ is combinatorial for every $i$ in $\mathcal{I}$. Then there exists a combinatorial model structure on the category of sections of $F$, denoted by $\operatorname{Sect}(\mathcal{I}, F)$ and called the injective model structure, such that a morphism of sections $\phi$ is a weak equivalence or a cofibration if and only if $\phi_{i}$ is a weak equivalence or a cofibration in $F(i)$ for every $i$ in $\mathcal{I}$, respectively. Moreover, if $F(i)$ is left or right proper for every $i \in \mathcal{I}$, then so is the model structure on $\operatorname{Sect}(\mathcal{I}, F)$.

Now, to model the homotopy limit of a left Quillen presheaf, we would like to construct a model structure on the category of sections whose cofibrant objects are precisely the levelwise cofibrant homotopy cartesian sections. This will be done by taking a right Bousfield localization of $\operatorname{Sect}(\mathcal{I}, F)$. The resulting model structure will be called the homotopy limit model structure.

The existence of the homotopy limit model structure when the category $\operatorname{Sect}(\mathcal{I}, F)$ is right proper was proved in [7, Theorem 3.2]. Without any properness assumptions, the homotopy limit model structure exists as a right model structure, as proved in [4, Theorem 5.25]. It follows directly from those results that if $F(i)$ is right proper for every $i$ in $\mathcal{I}$, then we get a full model structure. For the reader's convenience we spell this out in a little more detail.

Theorem 1.3. Let $F: \mathcal{I}^{\mathrm{op}} \rightarrow$ CAT be a left Quillen presheaf such that $F(i)$ is right proper and combinatorial for every $i$ in $\mathcal{I}$. Then there exists a combinatorial model structure on the category of sections of $F$, called the homotopy limit model structure, with the same fibrations as $\operatorname{Sect}(\mathcal{I}, F)$ and whose cofibrant objects are the sections that are cofibrant in $\operatorname{Sect}(\mathcal{I}, F)$ and homotopy cartesian.

Proof. Let $\mathcal{D}$ be the full subcategory of $\operatorname{Sect}(\mathcal{I}, F)$ consisting of the homotopy cartesian sections. Consider the functor

$$
\Phi: \operatorname{Sect}(\mathcal{I}, F) \longrightarrow \prod_{f: i \rightarrow j} \operatorname{Arr}(F(i))
$$

defined as $\Phi\left(\left(X_{i}\right)_{i \in \mathcal{I}}\right)=\prod_{f: i \rightarrow j} \varphi_{f}$, where $f$ runs over all morphisms of $\mathcal{I}$ and $\operatorname{Arr}(-)$ denotes the category of arrows, and let $Q$ denote an accessible cofibrant replacement functor in $\operatorname{Sect}(\mathcal{I}, F)$.

The categories $\operatorname{Sect}(\mathcal{I}, F)$ and $\prod_{f: i \rightarrow j} \operatorname{Arr}(F(i))$ are accessible (in fact, they are locally presentable; see [1, Corollary 1.54]) and the functor $\Phi$ is an accessible functor since it preserves all colimits (as these are computed levelwise). Hence $\Phi$ is an accessible functor between accessible categories.

Each $F(i)$ is combinatorial for every $i$ in $\mathcal{I}$, and hence by [23, Corollary A.2.6.6] the subcategory of weak equivalences weq $(F(i))$ is an accessible and accessibly embedded subcategory of $\operatorname{Arr}(F(i))$. Therefore, $\prod_{f: i \rightarrow j}$ weq $(F(i))$ is an accessible and accessibly embedded subcategory of $\prod_{f: i \rightarrow j} \operatorname{Arr}(F(i))$. 
By $\left[1\right.$, Remark 2.50], the preimage $(\Phi \circ Q)^{-1}\left(\prod_{f: i \rightarrow j}\right.$ weq $\left.(F(i))\right)$ is an accessible and accessibly embedded subcategory of $\operatorname{Sect}(\mathcal{I}, F)$. But this preimage is precisely $\mathcal{D}$.

Now, since $\mathcal{D}$ is accessible there exists a set $\mathcal{K}$ of objects and a regular cardinal $\lambda$ such that every object of $\mathcal{D}$ is a $\lambda$-filtered colimit (and hence a homotopy colimit if we choose $\lambda$ big enough; see [11, Proposition 7.3]) of objects in $\mathcal{K}$. Moreover, since $\mathcal{D}$ is accessibly embedded this homotopy colimit lies in $\mathcal{D}$.

The homotopy limit model structure is then the right Bousfield localization $R_{\mathcal{K}} \operatorname{Sect}(\mathcal{I}, F)$. (We can perform this right Bousfield localization because every $F(i)$ and hence $\operatorname{Sect}(\mathcal{I}, F)$ are right proper.) The fact that the cofibrant objects of this new model structure are precisely the levelwise cofibrant homotopy cartesian sections follows from [19, Theorem 5.1.5].

\section{Towers of Model Categories}

Let $\mathbb{N}$ be the category $0 \rightarrow 1 \rightarrow 2 \rightarrow \cdots$. A tower of model categories is a left Quillen presheaf $F: \mathbb{N}^{o p} \rightarrow$ CAT. The objects of the category of sections are then sequences $X_{0}, X_{1}, \ldots, X_{n}, \ldots$, where each $X_{i}$ is an object of $F(i)$, together with morphisms $\varphi_{i}: f^{*} X_{i+1} \rightarrow X_{i}$ in $F(i)$ for every $i \geq 0$, where $f: i \rightarrow i+1$ is the unique morphism from $i$ to $i+1$ in $\mathbb{N}$. A morphism between two sections $\phi_{\bullet}: X_{\bullet} \rightarrow Y_{\bullet}$ consists of morphisms $\phi_{i}: X_{i} \rightarrow Y_{i}$ in $F(i)$ such that the diagram

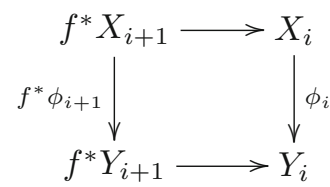

commutes for every $i \geq 0$.

Proposition 2.1. Let $F: \mathbb{N}$ op $\rightarrow$ CAT be a tower of model categories, where $F(i)$ is a combinatorial model category for every $i \geq 0$. There exists a combinatorial model structure on the category of sections, denoted by $\operatorname{Sect}(\mathbb{N}, F)$,

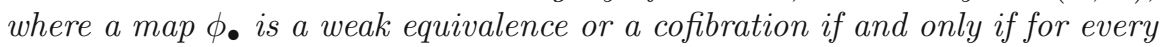
$i \geq 0$ the map $\phi_{i}$ is a weak equivalence or a cofibration in $F(i)$, respectively. The fibrations are the maps $\phi_{\bullet}: X_{\bullet} \rightarrow Y_{\bullet}$ such that $\phi_{0}$ is a fibration in $F(0)$ and

$$
X_{i+1} \longrightarrow Y_{i+1} \times_{f_{*} Y_{i}} f_{*} X_{i}
$$

is a fibration in $F(i+1)$ for every $i \geq 0$, where $f_{*}$ denotes the right adjoint to $f^{*}$. The fibrant objects are those sections $X_{\bullet}$ such that $X_{i}$ is fibrant in $F(i)$ and the morphism

$$
X_{i+1} \longrightarrow f_{*} X_{i}
$$

is a fibration in $F(i+1)$ for every $i \geq 0$.

Proof. The existence of the required model structure follows from Theorem 1.2. The description of the fibrations follows from [16, Theorem 3.1]. 
Proposition 2.2. Let $F: \mathbb{N}^{o p} \rightarrow$ CAT be a tower of model categories, where each $F(i)$ is combinatorial and right proper for every $i \geq 0$. Then there is a model structure $\operatorname{Tow}(F)$ on the category of sections of $F$ with the following properties:

(i) A morphism $\phi_{\bullet}$ is a fibration in $\operatorname{Tow}(F)$ if and only $\phi_{\bullet}$ is a fibration in $\operatorname{Sect}(\mathbb{N}, F)$.

(ii) A section $X_{\bullet}$ is cofibrant in $\operatorname{Tow}(F)$ if and only if $X_{i}$ is cofibrant in $F(i)$ and the morphism $f^{*} X_{i+1} \rightarrow X_{i}$ is a weak equivalence in $F(i)$ for every $i \geq 0$.

(iii) A morphism $\phi_{\bullet}$ between cofibrant sections is a weak equivalence in $\operatorname{Tow}(F)$ if and only if $\phi_{i}$ is a weak equivalence in $F(i)$ for every $i \geq 0$.

Proof. The existence of the model structure Tow $(F)$ follows from Theorem 1.3 applied to the left Quillen presheaf $F$. The characterization of the weak equivalences between cofibrant objects follows since $\operatorname{Tow}(F)$ is a right Bousfield localization of $\operatorname{Sect}(\mathbb{N}, F)$.

\subsection{Postnikov Sections of Model Structures}

Let $\mathcal{C}$ be a left proper combinatorial model category and $n \geq 0$. The model structure $P_{n} \mathcal{C}$ of $n$-types in $\mathcal{C}$ is the left Bousfield localization of $\mathcal{C}$ with respect to the set of morphisms $I_{\mathcal{C}} \square f_{n}$. Here $I_{\mathcal{C}}$ is the set of generating cofibrations of $\mathcal{C}, f_{n}: S^{n+1} \rightarrow D^{n+2}$ is the inclusion of simplicial sets from the $(n+1)$ sphere to the $(n+2)$-disk, and $\square$ denotes the pushout-product of morphisms constructed using the action of simplicial sets on $\mathcal{C}$ coming from the existence of framings; see [20, Section 5.4]. A longer account about model structures for $n$-types can be found in [18, Section 3].

For every $n<m$ the identity is a left Quillen functor $P_{m} \mathcal{C} \rightarrow P_{n} \mathcal{C}$. Thus we have a tower of model categories $P_{\bullet} \mathcal{C}: \mathbb{N}^{\circ p} \rightarrow$ CAT. The objects $X_{\bullet}$ of the category of sections are sequences

$$
\cdots \longrightarrow X_{n} \longrightarrow \cdots \longrightarrow X_{2} \longrightarrow X_{1} \longrightarrow X_{0}
$$

of morphisms in $\mathcal{C}$, and its morphisms $f_{\bullet}: X_{\bullet} \rightarrow Y_{\bullet}$ are given by commutative ladders

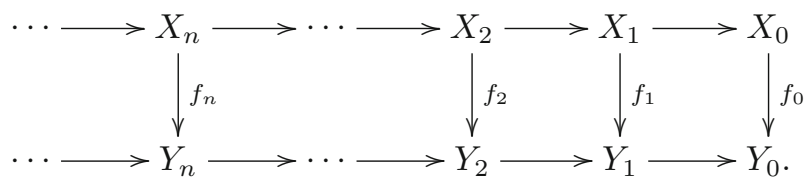

By Proposition 2.1, if $\mathcal{C}$ is a left proper combinatorial model category, then there exists a left proper combinatorial model structure on the category of sections $\operatorname{Sect}\left(\mathbb{N}, P_{\bullet} \mathcal{C}\right)$, where a map $f_{\bullet}$ is a weak equivalence or a cofibration if for every $n \geq 0$ the map $f_{n}$ is a weak equivalence or a cofibration in $P_{n} \mathcal{C}$, respectively. The fibrations are the maps $f_{\bullet}: X_{\bullet} \rightarrow Y_{\bullet}$ such that $f_{0}$ is a fibration in $P_{0} \mathcal{C}$ and

$$
X_{n} \longrightarrow Y_{n} \times_{Y_{n-1}} X_{n-1}
$$

is a fibration in $P_{n} \mathcal{C}$ for every $n \geq 1$. The fibrant objects can be characterized as follows: 
Lemma 2.3. Let $X_{\bullet}$ be a section of $P_{\bullet} \mathcal{C}$. The following are equivalent:

(i) $X_{\bullet}$ is fibrant in $\operatorname{Sect}\left(\mathbb{N}, P_{\bullet} \mathcal{C}\right)$.

(ii) $X_{0}$ is fibrant in $P_{0} \mathcal{C}$ and $X_{n+1} \rightarrow X_{n}$ is a fibration in $P_{n+1} \mathcal{C}$ for all $n \geq 0$.

(iii) $X_{n}$ is fibrant in $P_{n} \mathcal{C}$ and $X_{n+1} \rightarrow X_{n}$ is a fibration in $\mathcal{C}$ for all $n \geq 0$.

Proof. This follows because a fibration in $P_{n} \mathcal{C}$ is also a fibration in $P_{n+1} \mathcal{C}$ as well as a fibration in $\mathcal{C}$.

If the model structures for $n$-types $P_{n} \mathcal{C}$ are right proper for every $n \geq 0$, then by Proposition 2.2 the model structure $\operatorname{Tow}\left(P_{\bullet} \mathcal{C}\right)$ exists and will be denoted by $\operatorname{Post}(\mathcal{C})$. It has the following properties:

(i) A morphism $f_{\bullet}$ is a fibration in $\operatorname{Post}(\mathcal{C})$ if and only if $f_{\bullet}$ is a fibration in $\operatorname{Sect}\left(\mathbb{N}, P_{\bullet} \mathcal{C}\right)$.

(ii) A section $X_{\bullet}$ is cofibrant if and only if $X_{n}$ is cofibrant in $\mathcal{C}$ and $X_{n+1} \rightarrow X_{n}$ is a weak equivalence in $P_{n} \mathcal{C}$ for every $n \geq 0$.

(iii) A morphism $f_{\bullet}$ between cofibrant sections is a weak equivalence if and only if $f_{n}$ is a weak equivalence in $P_{n} \mathcal{C}$ for every $n \geq 0$.

For every $n \geq 0$ the identity functors form a Quillen pair id : $\mathcal{C} \rightleftarrows P_{n} \mathcal{C}$ : id, since $P_{n} \mathcal{C}$ is a left Bousfield localization of $\mathcal{C}$. This extends to a Quillen pair

$$
\text { id }: \mathcal{C}_{\mathrm{inj}}^{\mathbb{N}^{\mathrm{op}}} \rightleftarrows \operatorname{Sect}\left(\mathbb{N}, P_{\bullet} \mathcal{C}\right): \mathrm{id},
$$

where $\mathcal{C}_{\text {inj }}^{\mathbb{N}^{\text {op }}}$ denotes the category of $\mathbb{N}^{\text {op }}$-indexed diagrams with the injective model structure. Indeed weak equivalences and cofibrations in $\mathcal{C}_{\text {inj }}^{\mathbb{N}^{\text {op }}}$ are defined levelwise and every weak equivalence in $\mathcal{C}$ is a weak equivalence in $P_{n} \mathcal{C}$ for all $n \geq 0$. Hence, there is a Quillen pair

$$
\mathcal{C} \underset{\lim }{\stackrel{\text { const }}{\gtrless}} \mathcal{C}_{\text {inj }}^{\mathbb{N}^{\circ p}} \underset{\text { id }}{\gtrless} \operatorname{Sect}(\mathbb{N}, P \bullet \mathcal{C}) \stackrel{\text { id }}{\gtrless} \operatorname{id} \operatorname{Post}(\mathcal{C}),
$$

where const denotes the constant diagram functor.

Lemma 2.4. The adjunction const : $\mathcal{C} \rightleftarrows \operatorname{Post}(\mathcal{C})$ : $\lim$ is a Quillen pair.

Proof. By [19, Proposition 8.5.4(2)], it is enough to check that the left adjoint preserves trivial cofibrations and cofibrations between cofibrant objects. If $f$ is a trivial cofibration in $\mathcal{C}$ then const $(f)$ is a trivial cofibration in $\operatorname{Sect}\left(\mathbb{N}, P_{\bullet} \mathcal{C}\right)$. But since $\operatorname{Post}(\mathcal{C})$ is a right Bousfield localization of $\operatorname{Sect}\left(\mathbb{N}, P_{\bullet} \mathcal{C}\right)$ it has the same trivial cofibrations. Hence const $(f)$ is a trivial cofibration in $\operatorname{Post}(\mathcal{C})$.

Let $f: X \rightarrow Y$ be a cofibration between cofibrant objets in $\mathcal{C}$. Then const $(f)$ is a cofibration between cofibrant objects in $\operatorname{Sect}\left(\mathbb{N}, P_{\bullet} \mathcal{C}\right)$. But const $(X)$ and const $(Y)$ are both cofibrant in $\operatorname{Post}(\mathcal{C})$ by Proposition 2.2. Hence const $(f)$ is a cofibration in $\operatorname{Post}(\mathcal{C})$ if and only if it is a cofibration in $\operatorname{Sect}(\mathbb{N}, P \bullet \mathcal{C})$ (see $[19$, Proposition 3.3.16(2)]).

Let $\mathrm{sSet}_{*}$ denote the category of pointed simplicial sets with the KanQuillen model structure. Then the model structure Post $\left(\mathrm{sSet}_{*}\right)$ exists, since $P_{n}$ sSet $_{*}$ is right proper for every $n \geq 0$; see [10, Theorem 9.9]. 
Theorem 2.5. The Quillen pair const : $\operatorname{sSet}_{*} \rightleftarrows \operatorname{Post}\left(\mathrm{sSet}_{*}\right)$ : $\lim$ is a Quillen equivalence.

Proof. By [20, Proposition 1.3.13] it suffices to check that the derived unit and counit are weak equivalences. Let $X$ be a fibrant simplicial set. Then const $(X)$ is cofibrant in Post $\left(\operatorname{sSet}_{*}\right)$, since const is a left Quillen functor. Let

$$
\cdots \longrightarrow X_{n} \longrightarrow \cdots \longrightarrow X_{2} \longrightarrow X_{1} \longrightarrow X_{0}
$$

be a fibrant replacement of $\operatorname{const}(X)$ in Post( $\left.\operatorname{sSet}_{*}\right)$. Hence we have that $X_{n}$ is fibrant in $P_{n}$ sSet $_{*}$ and $X_{n+1} \rightarrow X_{n}$ is a fibration in sSet $_{*}$ and a weak equivalence in $P_{n}$ sSet $_{*}$ for all $n \geq 0$. By [15, Chap. VI, Theorem 3.5], the $\operatorname{map} X \rightarrow \lim X_{\bullet}$ is a weak equivalence.

Now, let $X_{\bullet}$ be any fibrant and cofibrant object in $\operatorname{Post}\left(\operatorname{sSet}_{*}\right)$. We have to see that the map const $\left(\lim X_{\bullet}\right) \rightarrow X_{\bullet}$ is a weak equivalence in Post $\left(\operatorname{sSet}_{*}\right)$. This is equivalent to seeing that the map $\lim X_{\bullet} \rightarrow X_{n}$ is a weak equivalence in $P_{n}$ sSet $_{*}$ for every $n \geq 0$. First note that since the category $\mathbb{N}_{>n}^{\text {op }}=\cdots \rightarrow$ $n+3 \rightarrow n+2 \rightarrow n+1$ is homotopy left cofinal in $\mathbb{N}^{o p}$ we have that $\lim X \bullet$ is weakly equivalent to $\lim _{\mathbb{N}_{>n}^{o p}} X_{\bullet}$ for every $n$ (see [19, Theorem 19.6.13]). Hence it is enough to check that the map $\lim _{\mathbb{N}_{>n}^{\text {op }}} X_{\bullet} \rightarrow X_{n}$ is a weak equivalence in $P_{n}$ sSet $_{*}$ for all $n \geq 0$. For every $n \geq 0$ we have a map of towers

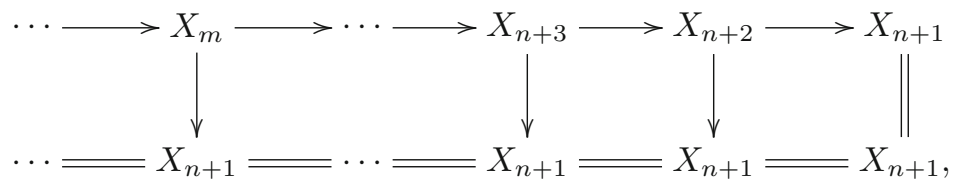

where each vertical map is a weak equivalence in $P_{n+1} \mathrm{sSet}_{*}$. Using the Milnor exact sequence (see [15, Chap. VI, Proposition 2.15]) we get a morphism of short exact sequences

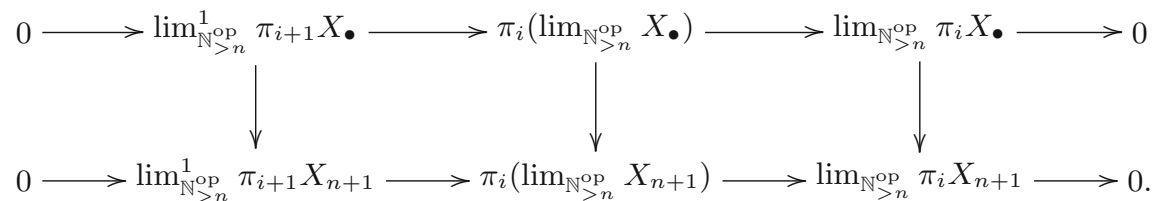

For $0 \leq i<n$ the left and right vertical morphisms are isomorphisms; hence the map $\lim _{\mathbb{N}_{>n}^{\text {op }}} X_{\bullet} \rightarrow X_{n+1}$ is a weak equivalence in $P_{n}$ sSet $_{*}$. Therefore, the map

$$
\lim _{\mathbb{N}_{>n}^{\text {op }}} X_{\bullet} \longrightarrow X_{n+1} \longrightarrow X_{n}
$$

is a weak equivalence in $P_{n}$ sSet $_{*}$ for $n \geq 0$.

Corollary 2.6. Let $X \rightarrow Y$ be a map in Post(sSet . $_{*}$. Then $X \rightarrow Y$ is a weak equivalence if and only if $\lim \widehat{X} \rightarrow \lim \widehat{Y}$ is a weak equivalence in sSet $_{*}$, where $\widehat{X}$ and $\widehat{Y}$ denote a fibrant replacement of $X$ and $Y$, respectively. 
Proof. We have the following commutative diagram:

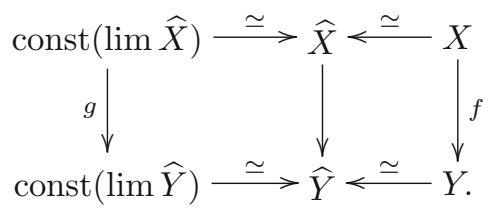

The horizontal arrows are weak equivalences because they are either a fibrant replacement or because the Quillen pair const and lim is a Quillen equivalence. So $f$ is a weak equivalence if and only if $g$ is a weak equivalence. But since const preserves and reflects weak equivalences between cofibrant objects (because it is the left adjoint of a Quillen equivalence), it follows that $g$ is a weak equivalence if and only if $\lim \widehat{X} \rightarrow \lim \widehat{Y}$ is a weak equivalence.

\subsection{Chromatic Towers of Localizations}

We can also use the homotopy limit model structure on towers of categories to obtain a categorified version of yet another classical result. The chromatic convergence theorem states that for a finite $p$-local spectrum $X$,

$$
X \simeq \operatorname{holim}_{n} L_{n} X,
$$

where $L_{n}$ denotes left localization at the chromatic homology theory $E(n)$ at a fixed prime $p$; see [26, Theorem 7.5.7]. The prime $p$ is traditionally omitted from notation. We will see that the Quillen adjunction between spectra and the left Quillen presheaf of chromatic localizations of spectra induces an adjunction between the homotopy category of finite spectra and the homotopy category of chromatic towers subject to a suitable finiteness condition. The chromatic convergence theorem then shows that the derived unit of this adjunction is a weak equivalence. By Sp in this section we always mean the category of $p$-local spectra symmetric spectra [21] and the prime $p$ will be fixed throughout the section.

Recall from [20, Section 6.1] that the homotopy category of a pointed model category supports a suspension functor with a right adjoint loop functor defined via framings. A model category is called stable if it is pointed and the suspension and loop operators are inverse equivalences on the homotopy category. Every combinatorial stable model category admits an enrichment over the category of symmetric spectra via stable frames; see [12,22].

Let $\mathcal{C}$ be a proper and combinatorial stable model category. Given a prime $p$, we define $L_{n} \mathcal{C}$ to be the left Bousfield localization of $\mathcal{C}$ with respect to the $E(n)$-equivalences, where $E(n)$ is considered at the prime $p$. By this, we mean Bousfield localisation at the set $I_{\mathcal{C}} \square \mathcal{S}_{E(n)}$, where $I_{\mathcal{C}}$ is the set of generating cofibrations of $\mathcal{C}$ and $\mathcal{S}_{E(n)}$ the generating acyclic cofibrations of $L_{E(n)} \mathrm{Sp}=L_{n} \mathrm{Sp}$. (The square denotes the pushout-product.) This defines a left Quillen presheaf

$$
L_{\bullet} \mathcal{C}: \mathbb{N}^{o p} \longrightarrow \mathrm{CAT} .
$$

By Proposition 2.1 we get the following: 
Proposition 2.7. There is a left proper, combinatorial and stable model structure on the category of sections $\operatorname{Sect}\left(\mathbb{N}, L_{\bullet} \mathcal{C}\right)$, such that a map is a weak equivalence or a cofibration if and only if each

$$
f_{n}: X_{n} \longrightarrow Y_{n}
$$

is a weak equivalence or a cofibration in $L_{n} \mathcal{C}$, respectively. $A$ map $f_{n}: X_{n} \rightarrow Y_{n}$ is a fibration if and only if $f_{0}$ is a fibration in $L_{0} \mathcal{C}$ and

$$
X_{n+1} \longrightarrow Y_{n+1} \times_{Y_{n}} X_{n}
$$

is a fibration in $L_{n+1} \mathcal{C}$ for all $n \geq 1$.

Note that the resulting model structure is stable as each $L_{n} \mathcal{C}$ is stable. We then perform a right Bousfield localization to obtain the homotopy limit model structure. Note that this again results in a stable model category [2, Proposition 5.6] as this right localization is stable in the sense of [2, Definition 5.3]. As left localization with respect to $E(n)$ is also stable in the sense of [2, Definition 4.2], $L_{n} \mathcal{C}$ is both left and right proper if $\mathcal{C}$ is; see [2, Propositions 4.6 and 4.7]. Hence, Proposition 2.2 implies the following result:

Proposition 2.8. Let $\mathcal{C}$ be a proper, combinatorial and stable model category. There is a model structure $\operatorname{Chrom}(\mathcal{C})$ on $\operatorname{Sect}\left(\mathbb{N}, L_{\bullet} \mathcal{C}\right)$ with the following properties:

(i) A morphism is a fibration in $\operatorname{Chrom}(\mathcal{C})$ if and only if it is a fibration in $\operatorname{Sect}\left(\mathbb{N}, L_{\bullet} \mathcal{C}\right)$.

(ii) An object $X_{\bullet}$ is cofibrant in $\operatorname{Chrom}(\mathcal{C})$ if and only if all the $X_{n}$ are cofibrant in $\mathcal{C}$ and $X_{n+1} \rightarrow X_{n}$ is an $E(n)$-equivalence for each $n$.

The following is useful to justify the name "homotopy limit model structure". Recall that Sp denotes here the category of $p$-local spectra.

Lemma 2.9. Let $f: X_{\bullet} \rightarrow Y_{\bullet}$ be a weak equivalence in $\operatorname{Chrom}(\mathrm{Sp})$. Then

$$
\operatorname{holim} X_{\bullet} \longrightarrow \operatorname{holim} Y_{\bullet}
$$

is a weak equivalence of spectra.

Proof. Let $f: X_{\bullet} \rightarrow Y_{\bullet}$ be a weak equivalence in Chrom(Sp). This implies that

$$
\operatorname{Ho}(\operatorname{Chrom}(\operatorname{Sp}))\left(\operatorname{const}(A), X_{\bullet}\right) \longrightarrow \operatorname{Ho}(\operatorname{Chrom}(\operatorname{Sp}))\left(\operatorname{const}(A), Y_{\bullet}\right)
$$

is an isomorphism for all cofibrant $A \in \mathrm{Sp}$. By Lemma 2.4, (const, lim) is a Quillen pair, so the above is equivalent to the claim that

$$
\left[A, \operatorname{holim} X_{\bullet}\right] \longrightarrow\left[A, \operatorname{holim} Y_{\bullet}\right]
$$

is an isomorphism for all cofibrant $A \in \mathcal{C}$, where the square brackets denote morphisms in the stable homotopy category. But as the class of all cofibrant spectra detects isomorphisms in the stable homotopy category, this is equivalent to

$$
\operatorname{holim} X_{\bullet} \longrightarrow \operatorname{holim} Y_{\bullet}
$$

being a weak equivalence of spectra as desired. 
Remark 2.10. It is important to note that we do not know if the converse is true. Looking at the proof of this lemma, we see that the following are equivalent:

(i) There is a set of objects of the form const $(G)$ in $\operatorname{Chrom}(\mathrm{Sp})$ that detect weak equivalences.

(ii) The weak equivalences in $\operatorname{Chrom}(\mathrm{Sp})$ are precisely the holimisomorphisms.

Unfortunately, it is not known from the definition of the homotopy limit model structure whether any of those equivalent conditions hold.

We can now turn to the main result of this subsection. For this, we need to specify our finiteness conditions. Recall that a $p$-local spectrum is called finite if it is in the full subcategory of the stable homotopy category Ho(Sp) which contains the sphere spectrum and is closed under exact triangles and retracts. We denote this full subcategory by $\operatorname{Ho}(\mathrm{Sp})^{\mathrm{fin}}$.

Definition 2.11. We call a diagram $X_{\bullet}$ in $\operatorname{Chrom}(\mathrm{Sp})$ finitary if holim $X_{\bullet}$ is a finite spectrum. By $\operatorname{Ho}(\operatorname{Chrom}(\mathrm{Sp}))^{F}$ we denote the full subcategory of the finitary diagrams in the homotopy category of $\operatorname{Chrom}(\mathrm{Sp})$.

Theorem 2.12. The Quillen adjunction const : Sp $\rightleftarrows \operatorname{Chrom}(\mathrm{Sp}): \lim$ induces an adjunction

$$
\mathrm{Ho}(\mathrm{Sp})^{\mathrm{fin}} \rightleftarrows \mathrm{Ho}(\mathrm{Chrom}(\mathrm{Sp}))^{F}
$$

and the derived unit is a weak equivalence.

Proof. First, we notice that the derived adjunction

$$
\text { Lconst : } \mathrm{Ho}(\mathrm{Sp}) \rightleftarrows \mathrm{Ho}(\operatorname{Chrom}(\mathrm{Sp})): \mathbb{R} \lim =\text { holim }
$$

restricts to an adjunction

$$
\mathbb{L} \text { const }: \operatorname{Ho}(\mathrm{Sp})^{\text {fin }} \rightleftarrows \operatorname{Ho}(\operatorname{Chrom}(\mathrm{Sp}))^{F}: \mathbb{R} \lim =\text { holim . }
$$

By definition, the homotopy limit of each finitary diagram is assumed to be a finite spectrum. On the other side,

$$
\operatorname{holim}(\mathbb{L} \operatorname{const}(X)) \simeq X
$$

is exactly the chromatic convergence theorem for finite spectra. The derived unit of the above adjunction is a weak equivalence. For a cofibrant spectrum

$$
X \longrightarrow\left(\operatorname{holim}(\operatorname{const}(X))=\operatorname{holim}_{n} L_{n} X\right)
$$

is again the chromatic convergence theorem.

We would really like to show that the above adjunction is an equivalence of categories, that is, that the counit is a weak equivalence, meaning that

$$
\operatorname{const}\left(\operatorname{holim} Y_{\bullet}\right) \longrightarrow Y_{\bullet}
$$

is a weak equivalence for $Y_{\bullet}$ a fibrant and cofibrant finitary diagram in Chrom(Sp). However, to show this we would need to know that the weak equivalences in Chrom(Sp) are exactly the holim-isomorphisms; see Remark 2.10. Furthermore, we would not just have to know that Chrom(Sp) 
has a constant set of generators but also that those generators are finitary, that is, the homotopy limit of each generator is finite.

\subsection{Convergence of Towers}

Let $\mathcal{C}$ be a left proper combinatorial model structure such that the model structures $P_{n} \mathcal{C}$ of $n$-types (see Sect. 2.1) are right proper, and hence the model structure $\operatorname{Post}(\mathcal{C})$ exists. In this section we are going to take a closer look at what it means for a tower in $\operatorname{Post}(\mathcal{C})$ to converge. Recall that we have a Quillen adjunction

$$
\text { const }: \mathcal{C} \rightleftarrows \operatorname{Post}(\mathcal{C}): \lim \text {. }
$$

The following terminology appears in [4, Definition 5.35].

Definition 2.13. The model category $\mathcal{C}$ is hypercomplete if the derived left adjoint of the previous Quillen adjunction is full and faithful, that is, if the composite

$$
\mathrm{Ho}(\mathcal{C}) \stackrel{\mathbb{L} \text { const }}{\longrightarrow} \mathrm{Ho}(\operatorname{Post}(\mathcal{C})) \stackrel{\text { holim }}{\longrightarrow} \mathrm{Ho}(\mathcal{C})
$$

is isomorphic to the identity.

We have seen in Sect. 2.1 that this is true for $\mathcal{C}=\operatorname{sSet}_{*}$. We have also seen in Theorem 2.12 that, under a finiteness assumption, the chromatic tower of spectra Chrom(Sp) is hypercomplete in this sense. We can also consider the case of left Bousfield localizations of $\operatorname{sSet}_{*}$, that is, $\mathcal{C}=L_{\mathcal{S}} \operatorname{sSet}_{*}$. In general, this model category will not be hypercomplete. Let $X$ be fibrant in $L_{\mathcal{S}}$ sSet $_{*}$, that is, fibrant as a simplicial set and $\mathcal{S}$-local. If we take a fibrant replacement of the constant tower const(Y) in $\operatorname{Post}\left(L_{\mathcal{S}} \operatorname{sSet}_{*}\right)$, we obtain a tower

$$
(\operatorname{const}(Y))^{\mathrm{fib}}=\left(\cdots \longrightarrow Y_{n} \longrightarrow Y_{n-1} \longrightarrow \cdots \longrightarrow Y_{0}\right)
$$

such that all the $Y_{i}$ are $\mathcal{S}$-local, $Y_{i}$ is $P_{i}$-local for all $i$ and $Y_{n} \rightarrow Y_{n-1}$ is a weak equivalence in $P_{n-1} L_{\mathcal{S}}$ sSet $_{*}$. However, this is not a fibrant replacement of const $(\mathrm{Y})$ in $\operatorname{Post}\left(\mathrm{sSet}_{*}\right)$, unless $L_{\mathcal{S}}$ commutes with all the localizations $P_{n}$. In this case, a Postnikov tower in $L_{\mathcal{S}} \operatorname{sSet}_{*}$ is also a Postnikov tower in $\mathrm{sSet}_{*}$, and hypercompleteness holds. This would be the case for $L_{\mathcal{S}}=L_{M R}$ for $R$ a subring of the rational numbers $\mathbb{Q}$, but it cannot be expected in general.

Let us recapture the classical case to get a more general insight into hypercompleteness. For $X$ in sSet $_{*}$ we know that $X \rightarrow \lim _{n} P_{n} X$ is a weak equivalence. This is equivalent to saying that for all $i$,

$$
\pi_{i}(X) \longrightarrow \pi_{i}\left(\lim _{n} P_{n} X\right)
$$

is an isomorphism of groups. But we have also seen that

$$
\pi_{i}\left(\lim _{n} P_{n} X\right)=\lim _{n} \pi_{i}\left(P_{n} X\right)
$$

as well as

$$
\pi_{i}\left(P_{n} X\right)=\left\{\begin{array}{cl}
\pi_{i}(X) & \text { if } i \leq n \\
0 & \text { if } i>n
\end{array}\right.
$$


Putting this together we get that, indeed, $\pi_{i}\left(\lim _{n} P_{n} X\right) \cong \pi_{i}(X)$ for all $i$. This is a special case of the following. A set of homotopy generators for a model category $\mathcal{C}$ consists of a small full subcategory $\mathcal{G}$ such that every object of $\mathcal{C}$ is weakly equivalent to a filtered homotopy colimit of objects of $\mathcal{G}$ and that by [11, Proposition 4.7] every combinatorial model category has a set of homotopy generators that can be chosen to be cofibrant. Let $\mathcal{C}$ be a proper combinatorial model category with a set of homotopy generators $\mathcal{G}$ and homotopy function complex $\operatorname{map}_{\mathcal{C}}(-,-)$. Then, for a cofibrant $X$, the map $X \rightarrow \operatorname{holim}_{n} P_{n} X$ is a weak equivalence in $\mathcal{C}$ if and only if

$$
\operatorname{map}_{\mathcal{C}}(G, X) \longrightarrow \operatorname{map}_{\mathcal{C}}\left(G, \operatorname{holim}_{n} P_{n} X\right)=\operatorname{holim}_{n} \operatorname{map}_{\mathcal{C}}\left(G, P_{n} X\right)
$$

is a weak equivalence in sSet for all $G \in \mathcal{G}$, where the equality holds by [19, Theorem 19.4.4(2)].

So from this we can see that if we had $\operatorname{map}_{\mathcal{C}}\left(G, P_{n} X\right) \cong P_{n} \operatorname{map}_{\mathcal{C}}(G, X)$ for all $G$ in $\mathcal{G}$, then we would get the desired weak equivalence because again

$$
\pi_{i} \operatorname{map}_{\mathcal{C}}\left(G, P_{n} X\right)=\pi_{i}\left(P_{n} \operatorname{map}_{\mathcal{C}}(G, X)\right) .
$$

We could also reformulate this statement by not using the full set of generators $\mathcal{G}$, since we are only making use of the fact that they detect weak equivalences.

Proposition 2.14. Let $h \mathcal{G}$ be a set in $\mathcal{C}$ that detects weak equivalences. If

$$
\operatorname{map}_{\mathcal{C}}\left(G, P_{n} X\right) \cong P_{n} \operatorname{map}_{\mathcal{C}}(G, X)
$$

for every $G$ in $h \mathcal{G}$, then $\mathcal{C}$ is hypercomplete.

We can follow this through with a non-simplicial example, bounded chain complexes of $\mathbb{Z}$-modules $\mathrm{Ch}_{b}(\mathbb{Z})$. Let us briefly recall Postnikov sections of chain complexes, which are discussed in detail in [18, Section 3.4]. As mentioned in Sect. 2.1, $P_{n} \mathrm{Ch}_{b}(\mathbb{Z})$ is the left Bousfield localization of $\mathrm{Ch}_{b}(\mathbb{Z})$ at

$$
W_{k}=I_{\mathrm{Ch}_{b}(\mathbb{Z})} \square\left\{f_{k}: S^{k+1} \longrightarrow D^{k+2}\right\} .
$$

The generating cofibrations of the projective model structure of $\mathrm{Ch}_{b}(\mathbb{Z})$ are the inclusions

$$
I_{\mathrm{Ch}_{b}(\mathbb{Z})}=\left\{\mathbb{S}^{n-1} \longrightarrow \mathbb{D}^{n} \mid n \geq 1\right\}
$$

where $\mathbb{S}^{n-1}$ is the chain complex which only contains $\mathbb{Z}$ in degree $n-1$ and is zero in all other degrees, and $\mathbb{D}^{n}$ is $\mathbb{Z}$ in degrees $n$ and $n-1$ with the identity differential and zero everywhere else. We can thus work out that

$$
W_{k}=\left\{\mathbb{S}^{n+k+1} \longrightarrow \mathbb{D}^{n+k+2} \mid n \geq 0\right\} .
$$

This means that a chain complex is a $k$-type if and only if its homology vanishes in degrees $k+1$ and above. The localization $M \longrightarrow P_{k} M$ is simply truncation above degree $k$. 
Let $\operatorname{Hom}(M, N)$ denote the mapping chain complex for $M, N$ in $\mathrm{Ch}_{b}(\mathbb{Z})$, that is,

$$
\operatorname{Hom}(M, N)_{k}=\prod_{i} \operatorname{Hom}_{\mathbb{Z}}\left(M_{i}, N_{i+k}\right)
$$

with differential $(d f)(x)=d(f(x))+(-1)^{k+1} f(d(x))$; see for example [20, Chap. 4.2]. We note that

$$
\pi_{i}\left(\operatorname{map}_{\mathrm{Ch}_{b}(\mathbb{Z})}(M, N)\right)=H_{i}(\operatorname{Hom}(M, N))
$$

because

$$
\begin{aligned}
\pi_{i}\left(\operatorname{map}_{\mathrm{Ch}_{b}(\mathbb{Z})}(M, N)\right) & =\left[S^{i}, \operatorname{map}_{\mathrm{Ch}_{b}(\mathbb{Z})}(M, N)\right]_{\mathrm{sSet}_{*}}=\left[M \otimes^{L} S^{i}, N\right]_{\mathrm{Ch}_{b}(\mathbb{Z})} \\
& =[M[i], N]_{\mathrm{Ch}_{b}(\mathbb{Z})}=[M \otimes \mathbb{Z}[i], N]_{\mathrm{Ch}_{b}(\mathbb{Z})} \\
& =[\mathbb{Z}[i], \operatorname{Hom}(M, N)]_{\mathrm{Ch}_{b}(\mathbb{Z})}=H_{i}(\operatorname{Hom}(M, N)) .
\end{aligned}
$$

So $\mathrm{Ch}_{b}(\mathbb{Z})$ is hypercomplete if $\operatorname{Hom}\left(G, P_{n} N\right)$ is quasi-isomorphic to $P_{n} \operatorname{Hom}(G, N)$ for all $G$ in $h \mathcal{G}$. For bounded below chain complexes, a set that detects weak equivalences can be taken to be

$$
h \mathcal{G}=\left\{\mathbb{S}^{i}=\mathbb{Z}[i] \mid i \geq 0\right\} .
$$

We have the following diagram of short exact sequences:

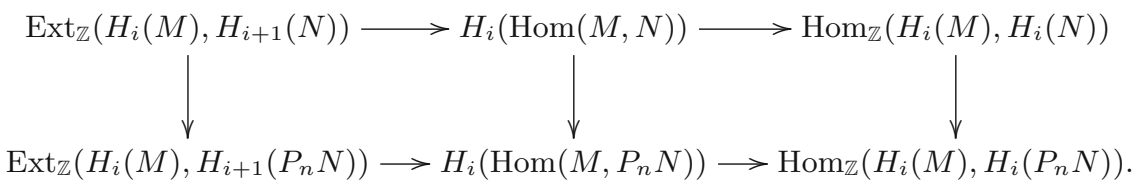

Using the 5-lemma we can read off that $H_{i}\left(\operatorname{Hom}\left(M, P_{n} N\right)\right)=0$ for $i>n$ as desired and that

$$
H_{i}\left(\operatorname{Hom}\left(M, P_{n} N\right)\right)=H_{i}(\operatorname{Hom}(M, N))
$$

for $i \leq n-1$, but unless $\operatorname{Ext}_{\mathbb{Z}}\left(H_{n}(M), H_{n+1}(N)\right)=0$ we do not get that

$$
H_{n}\left(\operatorname{Hom}\left(M, P_{n} N\right)\right)=H_{n}(\operatorname{Hom}(M, N)) .
$$

Note that in general it is not true that $\operatorname{Hom}\left(M, P_{n} N\right) \simeq P_{n} \operatorname{Hom}(M, N)$. However, as we only require the case $M=\mathbb{S}^{i}$, we have that

$$
\operatorname{Hom}\left(\mathbb{S}^{i}, N\right)=N[n],
$$

where $N[n]$ is the $n$-fold suspension of $N$. Thus,

$$
\operatorname{Hom}\left(G, P_{n} N\right)=P_{n} \operatorname{Hom}(G, N)
$$

for all $G$ in $h \mathcal{G}$, so $\mathrm{Ch}_{b}(\mathbb{Z})$ is hypercomplete as expected.

Remark 2.15. Another important example of a tower of model structures occurring in nature is given by the Taylor tower of Goodwillie calculus, where for every $n$ one considers the $n$-excisive model structure on the category of small endofunctors of simplicial sets; see [8, Section 4]. We do not discuss this example in this paper, and detailed relations to the aforementioned references could be a topic for future research. 


\section{Homotopy Fibered Products of Model Categories}

Let $\mathcal{I}$ be the small category

$$
1 \stackrel{\alpha}{\longleftarrow} 0 \stackrel{\beta}{\longrightarrow} 2 .
$$

A pullback diagram of model categories is a left Quillen presheaf $F: \mathcal{I}^{\text {op }} \rightarrow$ CAT. The objects $X_{\text {}}$ of the category of sections are given by three objects $X_{0}, X_{1}$ and $X_{2}$ in $F(0), F(1)$ and $F(2)$, respectively, together with morphisms

$$
\alpha^{*} X_{1} \longrightarrow X_{0} \longleftarrow \beta^{*} X_{2}
$$

in $F(0)$. A morphism $\phi_{\bullet}: X_{\bullet} \rightarrow Y_{\bullet}$ consists of morphisms $\phi_{i}: X_{i} \rightarrow Y_{i}$ in $F(i)$ for $i=0,1,2$, such that the diagram

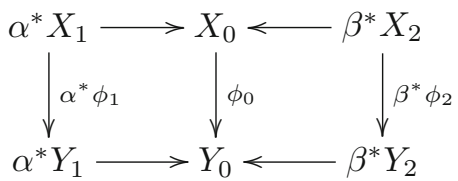

commutes.

Proposition 3.1. Let $F: \mathcal{I}^{\mathrm{op}} \rightarrow \mathrm{CAT}$ be a pullback diagram of model categories such that $F(i)$ is a combinatorial model category for every $i$ in $\mathcal{I}$. Then there exists a combinatorial model structure on the category of sections $\operatorname{Sect}(\mathcal{I}, F)$,

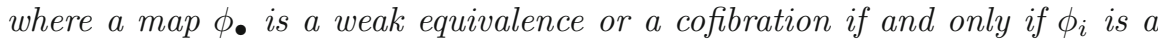
weak equivalence or cofibration in $F(i)$ for every $i$ in $\mathcal{I}$. The fibrations are the maps $\phi_{\bullet}: X_{\bullet} \rightarrow Y_{\bullet}$ such that $f_{0}$ is a fibration in $F(0)$ and

$$
X_{1} \longrightarrow Y_{1} \times_{\alpha_{*} Y_{0}} \alpha_{*} X_{0} \text { and } X_{2} \longrightarrow Y_{2} \times_{\beta_{*} Y_{0}} \beta_{*} X_{0}
$$

are fibrations in $F(1)$ and $F(2)$, respectively. In particular, $X_{\bullet}$ is fibrant if $X_{i}$ is fibrant in $F(i)$ and

$$
X_{1} \longrightarrow \alpha_{*} X_{0} \quad \text { and } \quad X_{2} \longrightarrow \beta_{*} X_{0}
$$

are fibrations in $F(1)$ and $F(2)$, respectively.

Proof. The existence of the required model structure follows from Theorem 1.2. The description of the fibrations follows from [16, Theorem 3.1].

Proposition 3.2. Let $F: \mathcal{I}^{\mathrm{op}} \rightarrow$ CAT be a pullback diagram of model categories such that $F(i)$ is combinatorial and right proper for every $i$ in $\mathcal{I}$. Then there is a model structure Fibpr $(F)$ on the category of sections of $F$, called the homotopy fibered product model structure, with the following properties:

(i) A morphism $\phi_{\bullet}$ is a fibration in $\operatorname{Fibpr}(F)$ if and only if $\phi_{\bullet}$ is a fibration in $\operatorname{Sect}(\mathcal{I}, F)$.

(ii) A section $X_{\bullet}$ is cofibrant in $\operatorname{Fibpr}(F)$ if and only if $X_{i}$ is cofibrant in $F(i)$ for every $i$ in $\mathcal{I}$ and the morphisms $\alpha^{*} X_{1} \rightarrow X_{0}$ and $\beta^{*} X_{2} \rightarrow X_{0}$ are weak equivalences in $F(0)$.

(iii) A morphism $\phi_{\bullet}$ between cofibrant sections is a weak equivalence if and only if $\phi_{i}$ is a weak equivalence in $F(i)$ for every $i$ in $\mathcal{I}$. 
Proof. The existence of the model structure $\operatorname{Fibpr}(F)$ follows from Theorem 1.3 applied to the left Quillen presheaf $F$. The characterization of the weak equivalences between cofibrant objects follows since $\operatorname{Fibpr}(F)$ is a right Bousfield localization of $\operatorname{Sect}(\mathcal{I}, F)$.

\subsection{Bousfield arithmetic Squares of Homological Localizations}

Let $\mathcal{C}$ be a left proper combinatorial stable model category and $E$ any spectrum. The model structure $L_{E} \mathcal{C}$ is the left Bousfield localization of $\mathcal{C}$ with respect to the set $I_{\mathcal{C}} \square \mathcal{S}_{E}$. Here $I_{\mathcal{C}}$ is the set of generating cofibrations of $\mathcal{C}$, the set $\mathcal{S}_{E}$ consists of the generating trivial cofibrations of the homological localization $L_{E} \mathrm{Sp}$, and $\square$ is the pushout-product defined via the action $\mathcal{C} \times \mathrm{Sp} \rightarrow \mathcal{C}$. This model structure is an example of a left Bousfield localization along a Quillen bifunctor, as studied in [18].

Now, let $J$ and $K$ be a partition of the set of prime numbers. By $\mathbb{Z}_{J}$ we denote the $J$-local integers, and by $M G$ the Moore spectrum of the group $G$. Consider the model structures $L_{M \mathbb{Z}_{J}} \mathcal{C}, L_{M \mathbb{Z}_{K}} \mathcal{C}$ and $L_{M \mathbb{Q}} \mathcal{C}$. Since, for every set of primes $P$, every $M \mathbb{Z}_{P}$-equivalence is an $M \mathbb{Q}$-equivalence, the identities $L_{M \mathbb{Z}_{J}} \mathcal{C} \rightarrow L_{M \mathbb{Q}} \mathcal{C}$ and $L_{M \mathbb{Z}_{K}} \mathcal{C} \rightarrow L_{M \mathbb{Q}} \mathcal{C}$ are left Quillen functors.

Thus we have a pullback diagram of model categories $L_{\bullet} \mathcal{C}: \mathcal{I}^{\text {op }} \rightarrow$ CAT, where $\mathcal{I}=1 \leftarrow 0 \rightarrow 2$ and $L_{0} \mathcal{C}=L_{M \mathbb{Q}} \mathcal{C}, L_{1} \mathcal{C}=L_{M \mathbb{Z}_{J}} \mathcal{C}$ and $L_{2} \mathcal{C}=L_{M \mathbb{Z}_{K}} \mathcal{C}$.

If $\mathcal{C}$ is a left proper combinatorial stable model category, then by Proposition 3.1 the model structure $\operatorname{Sect}\left(\mathcal{I}, L_{\bullet} \mathcal{C}\right)$ exists, and it is also a stable model structure because each of the involved model categories is stable.

Moreover, if in addition the model structures $L_{M \mathbb{Z}_{J}} \mathcal{C}, L_{M \mathbb{Z}_{K}} \mathcal{C}$ and $L_{M \mathbb{Q}} \mathcal{C}$ are right proper, then by Proposition 3.2 the model structure $\operatorname{Fibpr}\left(L_{\bullet} \mathcal{C}\right)$, which we denote by $\operatorname{Bou}(\mathcal{C})$, also exists. The model structure $\operatorname{Bou}(\mathcal{C})$ is also stable, since it is a right Bousfield localization with respect to a set of stable objects; see [2, Proposition 5.6].

Lemma 3.3. The adjunction const : $\mathcal{C} \rightleftarrows \operatorname{Bou}(\mathcal{C}): \lim$ is a Quillen pair.

Proof. The proof is the same as the one for Lemma 2.4.

Note that for any spectrum $E$, the model structure $L_{E} \mathrm{Sp}$ is right proper [2, Proposition 4.7]; hence the model structure Bou(Sp) exists.

Theorem 3.4. Let $\mathcal{C}$ be a proper and combinatorial stable model category. The Quillen pair const : $\mathcal{C} \rightleftarrows \operatorname{Bou}(\mathcal{C})$ : $\lim$ is a Quillen equivalence.

Proof. By [20, Proposition 1.3.13] it suffices to check that the derived unit and counit are weak equivalences.

Let $X$ be a fibrant and cofibrant object in $\mathcal{C}$. We need to show that

$$
X \longrightarrow \lim \left(\operatorname{const}(X)^{\mathrm{fib}}\right)
$$

is a weak equivalence in $\mathcal{C}$, where $(-)^{\text {fib }}$ denotes a fibrant replacement in $\operatorname{Bou}(\mathcal{C})$. The constant diagram const $(X)$ is cofibrant in $\operatorname{Bou}(\mathcal{C})$ since const is a left Quillen functor. Let

$$
L_{M \mathbb{Z}_{J}} X \longrightarrow L_{M \mathbb{Q}} X \longleftarrow L_{M \mathbb{Z}_{K}} X
$$


be a fibrant replacement of $\operatorname{const}(X)$ in $\operatorname{Bou}(\mathcal{C})$. We have that $L_{M_{\mathbb{Z}_{K}}} X$, $L_{M \mathbb{Z}_{J}} X$ and $L_{M \mathbb{Q}} X$ are fibrant in $L_{M \mathbb{Z}_{K}} \mathcal{C}, L_{M \mathbb{Z}_{J}} \mathcal{C}$ and $L_{M \mathbb{Q}} \mathcal{C}$, respectively, and the two maps are fibrations in $\mathcal{C}$ and weak equivalences in $L_{M \mathbb{Q}} \mathcal{C}$. Furthermore, the three localisations are smashing in Sp, so by [3, Lemma 6.7]

$$
L_{M \mathbb{Z}_{K}} X=X \wedge M \mathbb{Z}_{K}, L_{M \mathbb{Q}} X=X \wedge M \mathbb{Q} \text { and } L_{M \mathbb{Z}_{J}} X=X \wedge M \mathbb{Z}_{J}
$$

By [9, Proposition 2.10] we have that

$$
\lim \left(M \mathbb{Z}_{K} \longrightarrow M \mathbb{Q} \longleftarrow M \mathbb{Z}_{J}\right)=S,
$$

where $S$ denotes the sphere spectrum. Thus, the map

$$
\begin{aligned}
X & \longrightarrow \lim \left(L_{M \mathbb{Z}_{K}} X \longrightarrow L_{M \mathbb{Q}} X \longleftarrow L_{M \mathbb{Z}_{J}} X\right) \\
& =X \wedge \lim \left(M \mathbb{Z}_{K} \longrightarrow M \mathbb{Q} \longleftarrow M \mathbb{Z}_{J}\right)
\end{aligned}
$$

is a weak equivalence. The last equality follows because homotopy pullbacks commute with the action of spectra coming from framings, since in stable categories they are equivalent to homotopy pushouts.

Now, let $X \bullet$ be any fibrant and cofibrant object in $\operatorname{Bou}(\mathcal{C})$. We have to see that the map

$$
\operatorname{const}\left(\lim X_{\bullet}\right) \longrightarrow X_{\bullet}
$$

is a weak equivalence in $\operatorname{Bou}(\mathcal{C})$. This is equivalent to saying that the map $\lim X_{\bullet} \rightarrow X_{1}$ is a weak equivalence in $L_{M \mathbb{Z}_{J}} \mathcal{C}, \lim X_{\bullet} \rightarrow X_{2}$ is a weak equivalence in $L_{M \mathbb{Z}_{K}} \mathcal{C}$ and $\lim X_{\bullet} \rightarrow X_{12}$ is a weak equivalence in $L_{M \mathbb{Q}} \mathcal{C}$.

Note that if $A \rightarrow B$ is a weak equivalence in $L_{M \mathbb{Q}} \mathcal{C}, A$ is fibrant in $L_{M \mathbb{Z}_{K}} \mathcal{C}$ and $B$ is fibrant in $L_{M \mathbb{Q}} \mathcal{C}$, then $A \rightarrow B$ is a weak equivalence in $L_{M \mathbb{Z}_{J}} \mathcal{C}$. To see this, let $A \rightarrow L_{M \mathbb{Z}_{J}} A$ be a fibrant replacement of $A$ in $L_{M \mathbb{Z}_{J}} \mathcal{C}$. We are going to use [3, Lemma 6.7] again, which says that the weak equivalences in $L_{M \mathbb{Z}_{J}} \mathcal{C}$ are morphisms $f$ in $\mathcal{C}$ such that $f \wedge M \mathbb{Z}_{J}$ is a weak equivalence in $\mathcal{C}$. This makes the following argument the same as it would be for $\mathcal{C}=\mathrm{Sp}$.

Since $B$ is fibrant in $L_{M \mathbb{Q}} \mathcal{C}$, it is so in $L_{M \mathbb{Z}_{J}} \mathcal{C}$. Thus, there is a lifting

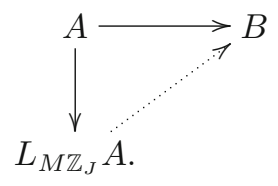

The left arrow is a weak equivalence in $L_{M \mathbb{Z}_{J}} \mathcal{C}$ and hence a weak equivalence in $L_{M \mathbb{Q}} \mathcal{C}$. Therefore, the dotted arrow is a weak equivalence in $L_{M \mathbb{Q}} \mathcal{C}$ between fibrant objects in $L_{M \mathbb{Q}} \mathcal{C}$. (Observe that $L_{M \mathbb{Z}_{J}} A$ is fibrant in $L_{M \mathbb{Z}_{J}} \mathcal{C}$ and $L_{M \mathbb{Z}_{K}} \mathcal{C}$ and hence in $L_{M \mathbb{Q}} \mathcal{C}$.) Thus, it is a weak equivalence in $\mathcal{C}$. This completes the proof of the claim since weak equivalences in $\mathcal{C}$ are weak equivalences in $L_{M \mathbb{Z}_{J}} \mathcal{C}$. 
Since $X_{\bullet}$ is fibrant and cofibrant, we have that in the pullback diagram

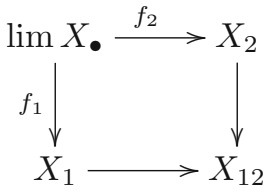

$X_{1}, X_{2}$ and $X_{12}$ are fibrant in $L_{M \mathbb{Z}_{J}} \mathcal{C}, L_{M \mathbb{Z}_{K}} \mathcal{C}$ and $L_{M \mathbb{Q}} \mathcal{C}$, respectively, and the right and bottom arrows are weak equivalences in $L_{M \mathbb{Q}} \mathcal{C}$ and fibrations in $L_{M \mathbb{Z}_{K}} \mathcal{C}$ and $L_{M \mathbb{Z}_{J}} \mathcal{C}$, respectively. By the previous observation and right properness of the model structures involved, the map $f_{1}: \lim X_{\bullet} \rightarrow X_{1}$ is a weak equivalence in $L_{M \mathbb{Z}_{J}}$, and $f_{2}: \lim X_{\bullet} \rightarrow X_{2}$ is a weak equivalence in $L_{M \mathbb{Z}_{K}} \mathcal{C}$, respectively. Thus, the map $\lim X_{\bullet} \rightarrow X_{12}$ is also a weak equivalence in $M \mathbb{Q}$, which means that const $\left(\lim X_{\bullet}\right) \longrightarrow X_{\bullet}$ is an objectwise weak equivalence, and thus a weak equivalence in $\operatorname{Bou}(\mathcal{C})$ as claimed.

Remark 3.5. There is a higher chromatic version of the objectwise statement. Here Sp denotes the category of $p$-local spectra. There is a homotopy fiber square

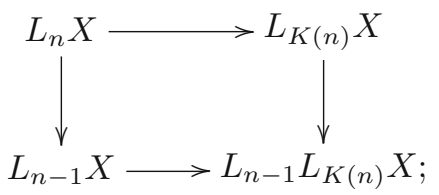

see [13, Section 3.9]. However, we cannot apply the methods of this section to get a result analogously to Theorem 3.4. This is due to the fact that $L_{K(n)} L_{n-1} \mathrm{Sp}$ is trivial as a model category. (By [25, Theorem 2.1], a spectrum is $E(n-1)$-local if and only if it is $K(i)$-local for $1 \leq i \leq n-1$. But the $K(n)$-localization of a $K(m)$-local spectrum is trivial for $n \neq m$.) Consider the homotopy fibered product model structure on

$$
L_{n-1} \mathrm{Sp} \longrightarrow L_{n-1} L_{K(n)} \mathrm{Sp} \longleftarrow L_{K(n)} \mathrm{Sp} .
$$

A fibrant and cofibrant diagram

$$
X_{1} \stackrel{f_{1}}{\longrightarrow} X_{0} \stackrel{f_{2}}{\longleftarrow} X_{2}
$$

would have to satisfy that $X_{1}$ is $E(n-1)$-local and $f_{1}$ is an $L_{n-1} L_{K(n)}$ localization. By the universal property of localizations, this means that $f_{1}$ factors over $L_{n-1} L_{K(n)} X_{1} \rightarrow X_{0}$. However, as $X_{1}$ is $E(n-1)$-local and thus $K(n)$-acyclic, this map (and thus $f_{1}$ ) is trivial. Thus we cannot reconstruct a pullback square like the above from this model structure.

\subsection{Homotopy Fibers of Localized Model Categories}

We will use the homotopy fibered product model structure to describe the homotopy fiber of Bousfield localizations. We can then use this to describe the layers of a Postnikov tower, among other examples.

Let $\mathcal{C}$ be a left proper pointed combinatorial model category and let $\mathcal{S}$ be a set of morphisms in $\mathcal{C}$. The identity $\mathcal{C} \rightarrow L_{\mathcal{S}} \mathcal{C}$ is a left Quillen functor and thus we have a pullback diagram of model categories $L_{\bullet} \mathcal{C}: \mathcal{I}^{\text {op }} \rightarrow \mathrm{CAT}$, 
where $\mathcal{I}=1 \leftarrow 0 \rightarrow 2$, and $L_{0}^{\mathcal{S}} \mathcal{C}=L_{\mathcal{S}} \mathcal{C}, L_{1}^{\mathcal{S}} \mathcal{C}=*$ and $L_{2}^{\mathcal{S}} \mathcal{C}=\mathcal{C}$. (Here * denotes the category with one object and one identity morphism with the trivial model structure.)

A section of $L_{\bullet}^{\mathcal{S}} \mathcal{C}$ is a diagram $* \rightarrow Y \leftarrow X$ in $\mathcal{C}$ where $*$ denotes the zero object. There is an adjunction

$$
\text { const }: \mathcal{C} \gtrless \operatorname{Sect}\left(\mathcal{I}, L_{\bullet} \mathcal{C}\right): e v_{2}
$$

where const $(X)=(* \rightarrow X \stackrel{1}{\leftarrow} X)$ and $e v_{2}(* \rightarrow Y \leftarrow X)=X$. We will denote $\operatorname{Fibpr}\left(L_{\bullet}^{\mathcal{S}}\right)$ by $\operatorname{Fib}\left(L_{\bullet}^{\mathcal{S}}\right)$ and we will call it the homotopy fiber of the Quillen pair $\mathcal{C} \rightleftarrows L_{\mathcal{S}} \mathcal{C}$.

Definition 3.6. Let $\mathcal{C}$ be a proper pointed combinatorial model category and let $\mathcal{K}$ be a set of objects and $\mathcal{S}$ be a set of morphisms in $\mathcal{C}$. We say that the colocalized model structure $C_{\mathcal{K}} \mathcal{C}$ and the localized model structure $L_{\mathcal{S}} \mathcal{C}$ are compatible when for every object $X$ in $\mathcal{C}, X$ is $\mathcal{K}$-colocal if and only if $X$ is cofibrant in $\mathcal{C}$ and the map $* \rightarrow X$ is an $\mathcal{S}$-local equivalence.

The stable case is discussed in detail in [2, Section 10] where such model structures are called "orthogonal"; see also Sect. 3.5.

Remark 3.7. Note that if $C_{\mathcal{K}} \mathcal{C}$ and $L_{\mathcal{S}} \mathcal{C}$ are compatible, then it follows from the definitions that $* \rightarrow Y \leftarrow X$ is cofibrant in $\operatorname{Fib}\left(L_{\bullet}^{\mathcal{S}} \mathcal{C}\right)$ if and only if both $X$ and $Y$ are $\mathcal{K}$-colocal and cofibrant in $\mathcal{C}$. If $* \rightarrow Y \leftarrow X$ is moreover fibrant in $\operatorname{Fib}\left(L_{\bullet}^{\mathcal{S}} \mathcal{C}\right)$, then $Y$ is weakly contractible since $Y$ is $\mathcal{S}$-local and $* \rightarrow Y$ is an $\mathcal{S}$-equivalence and $X \rightarrow Y$ is a fibration in $\mathcal{C}$.

Theorem 3.8. Let $\mathcal{C}$ be a proper pointed combinatorial model category and let $\mathcal{K}$ be a set of objects and $\mathcal{S}$ be a set of morphisms in $\mathcal{C}$. If $C_{\mathcal{K}} \mathcal{C}$ and $L_{\mathcal{S}} \mathcal{C}$ are compatible, then the adjunction

$$
\text { const }: C_{\mathcal{K}} \mathcal{C} \rightleftarrows \operatorname{Fib}\left(L_{\bullet}^{\mathcal{S}} \mathcal{C}\right): e v_{2}
$$

is a Quillen equivalence.

Proof. We will first show that the adjunction is a Quillen pair. By [19, Propostion 8.5.4(2)], it is enough to check that the left adjoint preserves trivial cofibrations and sends cofibrations between cofibrant objects to cofibrations.

Let $f$ be a trivial cofibration in $C_{\mathcal{K}} \mathcal{C}$. Then $f$ is a trivial cofibration in $\mathcal{C}$ and, therefore, const $(f)$ is a trivial cofibration in $\operatorname{Sect}\left(\mathcal{I}, L_{\bullet} \mathcal{C}\right)$ and thus a trivial cofibration in $\operatorname{Fib}\left(L_{\bullet}^{\mathcal{S}} \mathcal{C}\right)$.

Now let $f: X \rightarrow Y$ be a cofibration between cofibrant objects in $C_{\mathcal{K}} \mathcal{C}$. Then $f$ is a cofibration between cofibrant objects in $\mathcal{C}$ and hence const $(f)$ is also a cofibration between cofibrant objects in $\operatorname{Sect}\left(\mathcal{I}, L_{\bullet} \mathcal{C}\right)$. But $\operatorname{const}(X)$ and const $(Y)$ are cofibrant in $\operatorname{Fib}\left(L_{\bullet}^{\mathcal{S}} \mathcal{C}\right)$, since $C_{\mathcal{K}} \mathcal{C}$ and $L_{\mathcal{S}} \mathcal{C}$ are compatible and, therefore, the maps $* \rightarrow X$ and $* \rightarrow Y$ are $\mathcal{S}$-local equivalences. Hence const $(f)$ is a cofibration in $\operatorname{Fib}\left(L_{\bullet} \mathcal{C}\right)$, by [19, Proposition 3.3.16(2)].

To prove that it is a Quillen equivalence, it suffices to show that the derived unit and counit are weak equivalences; see [20, Proposition 1.3.13]. Let 
$X$ be a cofibrant object in $C_{\mathcal{K}} \mathcal{C}$. Then we can construct a fibrant replacement for $\operatorname{const}(X)$ in $\operatorname{Fib}\left(L_{\bullet} \mathcal{C}\right)$ as follows:

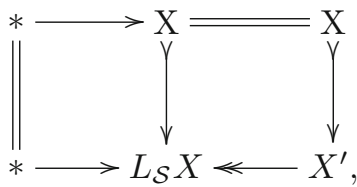

where the map $X \rightarrow L_{\mathcal{S}} X$ is a trivial cofibration in $L_{\mathcal{S}} \mathcal{C}$ and $X \rightarrow X^{\prime} \rightarrow L_{\mathcal{S}} X$ is a factorization in $\mathcal{C}$ of the previous map as a trivial cofibration followed by a fibration. Indeed, the map between the two sections is a trivial cofibration in $\operatorname{Fib}\left(L_{\bullet}^{\mathcal{S}} \mathcal{C}\right)$ since it is a levelwise trivial cofibration, and $* \rightarrow L_{\mathcal{S}} X \leftarrow X^{\prime}$ is fibrant in $\operatorname{Fib}\left(L_{\bullet}^{\mathcal{C}}\right)$ since $L_{\mathcal{S}} X$ is fibrant in $L_{\mathcal{S}} \mathcal{C}, X^{\prime}$ is fibrant in $\mathcal{C}$ and $X^{\prime} \rightarrow L_{\mathcal{S}} X$ is a fibration in $\mathcal{C}$.

Therefore, the map $X \rightarrow e v_{2}(\operatorname{const}(X)) \rightarrow e v_{2}(R(\operatorname{const}(X)))$, where $R$ denotes fibrant replacement in $\operatorname{Fib}\left(L_{\bullet}^{\mathcal{S}} \mathcal{C}\right)$, is precisely the map $X \rightarrow X^{\prime}$, which is a weak equivalence in $C_{\mathcal{K}} \mathcal{C}$ since it was already a weak equivalence in $\mathcal{C}$.

Finally, let $* \rightarrow Y \leftarrow X$ be a fibrant and cofibrant section in $\operatorname{Fib}\left(L_{\bullet}^{\mathcal{S}} \mathcal{C}\right)$. We need to check that the composite

$\operatorname{const}\left(Q\left(e v_{2}(* \rightarrow Y \leftarrow X)\right)\right) \longrightarrow \operatorname{const}\left(e v_{2}(* \rightarrow Y \leftarrow X)\right) \longrightarrow(* \rightarrow Y \leftarrow X)$

is a weak equivalence in $\operatorname{Fib}\left(L_{\bullet}^{\mathcal{S}} \mathcal{C}\right)$. But $e v_{2}(* \rightarrow Y \leftarrow X)=X$ is already cofibrant in $C_{\mathcal{K}} \mathcal{C}$, by Remark 3.7. Therefore, we need to show that the map of sections

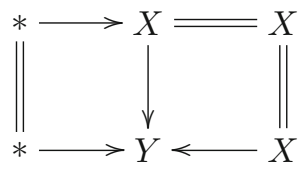

is a weak equivalence in $\operatorname{Fib}\left(L_{\bullet}^{\mathcal{S}}\right)$. Since both sections are cofibrant, it is enough to see that the map in the middle is a weak equivalence in $L_{\mathcal{S}} \mathcal{C}$, which follows again from Remark 3.7.

\subsection{Postnikov Sections and Connective Covers of Simplicial Sets}

We can use this setup to describe the "layers" of Postnikov towers. Let sSet $*$ denote the category of pointed simplicial sets. Consider the model structure $P_{k}$ sSet $_{*}=L_{\mathcal{S}} \mathrm{sSet}_{*}$ for $k$-types, that is, the left Bousfield localization of sSet $_{*}$ with respect to the set of inclusions $\mathcal{S}=\left\{S^{k+1} \rightarrow D^{k+2}\right\}$. If $\mathcal{K}=$ $\left\{S^{k+1}\right\}$, then the right Bousfield localization $C_{k} \operatorname{sSet}_{*}=C_{\mathcal{K}} \mathrm{sSet}_{*}$ is the model structure for $k$-connective covers, and $P_{k} \mathrm{sSet}_{*}$ and $C_{k} \mathrm{sSet}_{*}$ are compatible, since for every $X$ there is a fiber sequence

$$
C_{k} X \longrightarrow X \longrightarrow P_{k} X
$$

where $C_{k} X$ denotes the $k$ th connective cover of $X$. By Theorem 3.8 the model categories $C_{k} \mathrm{sSet}_{*}$ and $\operatorname{Fib}\left(L_{\bullet}^{\mathcal{S}} \mathrm{sSet}_{*}\right)$ are Quillen equivalent. 
Let $\mathcal{S}=\left\{S^{n+1} \rightarrow D^{n+2}\right\}$ and $\mathcal{K}=\left\{S^{n+1}\right\}$, as before, and let $\mathcal{C}$ be a proper combinatorial model category. Then we define $L_{\mathcal{S}} \mathcal{C}$ as the left Bousfield localization of $\mathcal{C}$ with respect to the set $I_{\mathcal{C}} \square \mathcal{S}$ and $C_{\mathcal{K}} \mathcal{C}$ as the right Bousfield localization of $\mathcal{C}$ with respect to $\mathcal{G}_{\mathcal{C}} \otimes \mathcal{K}$. Here $I_{\mathcal{C}}$ is the set of generating cofibrations of $\mathcal{C}, \mathcal{G}_{\mathcal{C}}$ is a set of homotopy generators, $\otimes$ denotes the simplicial action given by a framing and $\square$ the pushout product. A fuller account of localized model structures along Quillen bifunctors can be found in [18]. In general, $L_{\mathcal{S}} \mathcal{C}$ and $C_{\mathcal{K}} \mathcal{C}$ are not necessarily compatible, so Theorem 3.8 will not hold in this case for arbitrary $\mathcal{C}$. However, examples where compatibility holds include the category of chain complexes $\mathrm{Ch}_{b}(R)$ and stable localizations; see Sect. 3.5. fibration

We can also consider $\operatorname{Fib}\left(L_{\bullet}^{\mathcal{S}} P_{k+1} \mathrm{sSet}_{*}\right)$. Since for every $X$ we have a

$$
K\left(\pi_{k+1} X, k+1\right) \longrightarrow P_{k+1} X \longrightarrow P_{k} X,
$$

the model structures $C_{k} P_{k+1} \mathrm{sSet}_{*}$ and $P_{k} P_{k+1} \mathrm{sSet}_{*}=P_{k} \mathrm{sSet}_{*}$ are compatible. Hence Theorem 3.8 directly implies

Corollary 3.9. The model structures $C_{k} P_{k+1} \operatorname{sSet}_{*}$ and $\operatorname{Fib}\left(L_{\bullet}^{\mathcal{S}} P_{k+1} \operatorname{sSet}_{*}\right)$ are Quillen equivalent.

This means that we can view $C_{k} P_{k+1} \operatorname{sSet}_{*}$ as the $k$ th layer of the Postnikov tower model structure. Note that $\operatorname{Ho}\left(C_{k} P_{k+1} \mathrm{sSet}_{*}\right)$ is equivalent to the category of abelian groups for $k \geq 1$.

\subsection{Nullifications and Cellularizations of Spectra}

Let $\mathrm{Sp}$ be a suitable model structure for the category of spectra, for instance, symmetric spectra and let $\mathcal{S}$ be a single map $E \rightarrow *$. Then $L_{\mathcal{S}} \mathrm{Sp}=P_{E} \mathrm{Sp}$ is called the $E$-nullification of $\mathrm{Sp}$ and $C_{E} \mathrm{Sp}$ is called the E-cellularization of Sp. As follows from [17, Theorem 3.6] we have the following compatibility between localized and colocalized model structures:

(i) If the induced map $\operatorname{Ho}(\mathrm{Sp})\left(\Sigma^{-1} E, C_{E} X\right) \rightarrow \operatorname{Ho}(\mathrm{Sp})\left(\Sigma^{-1} E, X\right)$ is injective for every $X$, then $C_{E}$ Sp and $P_{E}$ Sp are compatible.

(ii) If the induced map $\operatorname{Ho}(\mathrm{Sp})(E, X) \rightarrow \operatorname{Ho}(\mathrm{Sp})\left(E, P_{\Sigma E} X\right)$ is the zero map for every $X$, then $C_{E}$ Sp and $P_{\Sigma E}$ Sp are compatible.

\subsection{Stable Localizations and Colocalizations}

Let $\mathcal{C}$ be a proper combinatorial stable model category and let $\mathcal{G}_{\text {Sp }}$ denote a set of cofibrant homotopy generators for the model category of symmetric spectra Sp. Recall that a set of homotopy generators for a model category $\mathcal{C}$ consists of a small full subcategory $\mathcal{G}_{\mathcal{C}}$ such that every object of $\mathcal{C}$ is weakly equivalent to a filtered homotopy colimit of objects of $\mathcal{G}_{\mathcal{C}}$ and that by $[11$, Proposition 4.7] every combinatorial model category has a set of homotopy generators that can be chosen to be cofibrant.

A set of maps $\mathcal{S}$ in a stable model category is said to be stable if the class of $\mathcal{S}$-local objects is closed under suspension. Let $\mathcal{S}$ be a stable set of morphisms in $\mathcal{C}$ and let $\mathcal{K}=\operatorname{cof}(\mathcal{S})$ be the set of cofibers of the elements of $\mathcal{S}$. Then we have that $\operatorname{cof}\left(\mathcal{S} \otimes \mathcal{G}_{\mathrm{Sp}}\right)=\operatorname{cof}(\mathcal{S}) \otimes \mathcal{G}_{\mathrm{Sp}}=\mathcal{K} \otimes \mathcal{G}_{\text {Sp }}$, where 
denotes the action of $\mathrm{Sp}$ on $\mathcal{C}$. Hence, by [2, Proposition 10.3] it follows that $L_{\mathcal{S} \otimes \mathcal{G}_{\mathrm{Sp}}} \mathcal{C}$ and $C_{\mathcal{K} \otimes \mathcal{G}_{\mathrm{Sp}}} \mathcal{C}$ are compatible. Therefore, Theorem 3.8 readily implies the following fact:

Corollary 3.10. The model categories $C_{\mathcal{K} \otimes \mathcal{G}_{\mathrm{Sp}}} \mathcal{C}$ and $\mathrm{Fib}\left(L_{\bullet}^{\mathcal{S} \otimes \mathcal{G}_{\mathrm{Sp}}} \mathcal{C}\right)$ are Quillen equivalent.

\section{Acknowledgments}

J. J. Gutiérrez would like to thank Dimitri Ara for many useful conversations on some of the topics of this paper, and Ieke Moerdijk for suggesting the idea of studying towers of localized model structures. C. Roitzheim would like to thank David Barnes for motivating discussions and the Radboud Universiteit Nijmegen for their hospitality. Both authors thank the referee and the associate editor for very useful comments that helped improving the contents and presentation of the paper.

Open Access. This article is distributed under the terms of the Creative Commons Attribution 4.0 International License (http://creativecommons.org/licenses/ by/4.0/), which permits unrestricted use, distribution, and reproduction in any medium, provided you give appropriate credit to the original author(s) and the source, provide a link to the Creative Commons license, and indicate if changes were made.

\section{References}

[1] Adámek, J., Rosický, J.: Locally Presentable and Accessible Categories, London Mathematical Society Lecture Note Series, vol. 189. Cambridge University Press, Cambridge (1994)

[2] Barnes, D., Roitzheim, C.: Stable left and right Bousfield localisations. Glasg. Math. J. 56(1), 13-42 (2014)

[3] Barnes, D., Roitzheim, C.: Homological localisation of model categories. Appl. Categ. Struct. 23(3), 487-505 (2015)

[4] Barwick, C.: On left and right model categories and left and right Bousfield localizations. Homol. Homotopy Appl. 12(2), 245-320 (2010)

[5] Beke, T.: Sheafifiable homotopy model categories. Math. Proc. Camb. Philos. Soc. 129(3), 447-475 (2000)

[6] Bergner, J: Homotopy fiber products of homotopy theories. Isr. J. Math. 185, 389-411 (2011)

[7] Bergner, J.: Homotopy limits of model categories and more general homotopy theories. Bull. Lond. Math. Soc. 44(2), 311-322 (2013)

[8] Biedermann, G., Chorny, B., Röndigs, O.: Calculus of functors and model categories. Adv. Math. 214, 92-115 (2007)

[9] Bousfield, A.K.: The localization of spectra with respect to homology. Topology 18(4), 257-281 (1979)

[10] Bousfield, A.K.: On the telescopic homotopy theory of spaces. Trans. Am. Math. Soc. 353(6), 2391-2426 (2001) 
[11] Dugger, D.: Combinatorial model categories have presentations. Adv. Math. 164, 177-201 (2001)

[12] Dugger, D: Spectral enrichments of model categories. Homol. Homotopy Appl. 8(1), 1-30 (2006)

[13] Dwyer, W.G.: Localizations, Axiomatic, Enriched and Motivic Homotopy Theory, 3-28, NATO Science Series II: Mathematics, Physics and Chemistry, vol. 131. Kluwer Academic Publications, Dordrecht (2004)

[14] Gabriel, P., Ulmer, F.: Lokal präsentierbare Kategorien. Lecture Notes in Mathematics, vol. 221. Springer, Berlin (1971)

[15] Goerss, P.G., Jardine, J.F.: Simplicial Homotopy Theory. Progress in Mathematics, vol. 174. Birkhäuser, Basel (1999)

[16] Greenlees, J.P.C., Shipley, B.: Homotopy theory of modules over diagrams of rings. Proc. Am. Math. Soc. Ser. B 1, 89-104 (2014)

[17] Gutiérrez, J.J.: Cellularization of structures in stable homotopy categories. Math. Proc. Camb. Philos. Soc. 153, 399-418 (2012)

[18] Gutiérrez, J.J., Roitzheim, C.: Bousfield Localisations Along Quillen Bifunctors (2014) arXiv:1411.0500

[19] Hirschhorn, P.S.: Model Categories and Their Localizations. Mathematical Surveys and Monographs, vol. 99. American Mathematical Society, Providence (2003)

[20] Hovey, M.: Model Categories. Mathematical Surveys and Monographs, vol. 63. American Mathematical Society, Providence (1999)

[21] Hovey, M., Shipley, B., Smith, J.H.: Symmetric spectra. J. Am. Math. Soc. 13(1), 149-208 (2000)

[22] Lenhardt, F.: Stable frames in model categories. J. Pure Appl. Algebra 216(5), 1080-1091 (2012)

[23] Lurie, J.: Higher Topos Theory. Annals of Mathematical Studies, vol. 170. Princeton University Press, Princeton (2009)

[24] Makkai, M., Paré, R.: Accessible Categories: The Foundations of Categorical Model Theory. Contemporary Mathematics, vol. 104. American Mathematical Society, Providence (1989)

[25] Ravenel, D.C.: Localization with respect to certain periodic homology theories. Am. J. Math. 106(2), 351-414 (1984)

[26] Ravenel, D.C.: Nilpotence and Periodicity in Stable Homotopy Theory. Annals of Mathematical Studies, vol. 128. Princeton University Press, Princeton (1992)

[27] Toën, B.: Derived Hall algebras. Duke Math. J. 135(3), 587-615 (2006)

Javier J. Gutiérrez

Institute for Mathematics, Astrophysics and Particle Physics

Radboud Universiteit Nijmegen

Heyendaalseweg 135

6525 AJ Nijmegen

The Netherlands

e-mail: j.gutierrez@math.ru.nl

URL: http://www . math.ru.nl/gutierrez 
Constanze Roitzheim

School of Mathematics

Statistics and Actuarial Science

University of Kent

Canterbury, Kent CT2 7NF

UK

e-mail: c.roitzheim@kent.ac.uk

URL: http://www.kent.ac.uk/smsas/personal/csrr

Received: July 20, 2015.

Revised: March 4, 2016.

Accepted: March 30, 2016. 